\title{
A Prospective Study of Maintenance Electroconvulsive Therapy in an Elderly Depressed Population
}

\author{
Popuri Krishna',2, Lakyntiew Aulakh ${ }^{1,2}$, Declan Boylan',2, Louis Lakatos ${ }^{3}$ \\ ${ }^{1}$ Department of Psychiatry, Health Sciences North, Sudbury, Canada \\ ${ }^{2}$ Northern Ontario School of Medicine, Laurentian University, Sudbury, Canada \\ ${ }^{3}$ Health Sciences North Research Institute, Sudbury, Canada \\ Email: pkrishna@hsnsudbury.ca
}

How to cite this paper: Krishna, P., Aulakh, L., Boylan, D. and Lakatos, L. (2021) A Prospective Study of Maintenance Electroconvulsive Therapy in an Elderly Depressed Population. Open Journal of Psychiatry, 11, 63-70.

https://doi.org/10.4236/ojpsych.2021.112006

Received: November 30, 2020

Accepted: March 7, 2021

Published: March 10, 2021

Copyright $\odot 2021$ by author(s) and Scientific Research Publishing Inc. This work is licensed under the Creative Commons Attribution International License (CC BY 4.0).

http://creativecommons.org/licenses/by/4.0/

\begin{abstract}
Objective: This study was conducted to discern the efficacy of maintenance electroconvulsive therapy (M-ECT) in a population of depressed elderly individuals with treatment-resistant depression. Methodology: Twenty-nine ( $\mathrm{N}$ =29) individuals over the age of 65 years of age and older were assigned to a control or treatment group on the basis of their decision to receive M-ECT (treatment group) or to refrain from receiving the treatment (control group). A battery of psychometric tests designed to measure severity of depression, quality of life, and cognition were administered at baseline as well as at 6-month and 1-year intervals. Results: Statistical analysis of the data indicated no significant differences in the efficacy of M-ECT between the control and treatment groups in any of the tests administered during the participation of the study. Conclusion: The results of the study suggest that there is no added benefit for patients administered M-ECT. However, study sample size and availability of alternative treatment regimens for the control group limit generalizability of these findings and warrant further investigation.
\end{abstract}

\section{Keywords}

Maintenance Electroconvulsive Therapy (M-ECT), Depression, Elderly Population

\section{Introduction}

Elderly patients in treatment for severe depressive disorder are often treated with a combination of pharmacotherapy and psycho-social interventions. However, the efficacy of these treatments used alone often wanes as the patients become 
resistant to them. In addition medical problems, drug interactions, and poor tolerance to pharmacotherapy complicate the management for successful remission of depression in the elderly population [1]. Electro Convulsive Therapy (ECT) has been used safely within the elderly population with improvement in depression [2] and is often a preferred option for elderly patients with underlying medical conditions or low tolerance to pharmacologicals [3].

There are earlier studies looking at the role of maintenance ECT in the management of severe depression in the elderly. The majority of the available literature assumes the form of either case studies or retrospective studies [3]. A recent prospective study was conducted by Kellner et al. in 2006 [4]; however, its duration was only six months. The current body of knowledge is sparse on the incorporation of maintenance-ECT (M-ECT) in the long-term and in combination with a standard maintenance pharmacological regime, though it is recognized as a valuable and underutilized method of treatment [5].

While ECT use decreased in the late 80's and early 90's, its use has been increasing in recent years presumably because since it is safe, works well, and has a rapid response with little side effects [6]. The most common diagnostic indication for ECT is major depression [7]. James Tew et al. [8] reported that elderly depressed patients tolerated ECT in a manner similar to younger patients and demonstrated a similar or better acute response. Further, in elderly patients treated with ECT, the occurrences of relapses were fewer and those that did experience relapses required fewer hospital days during their treatments [9]. Side effects in the elderly were generally minimal with the most frequent occurrence being some short-term memory loss that was generally regained within a few days post ECT treatment.

As ECT methodology has become more refined, its use in older adults has steadily grown. Thompson et al. [10] reported that in 1986 approximately one third of people receiving ECT were aged 65 or older. It has also been suggested that rather than diminishing effects, ECT is more effective and beneficial in elderly patients than in the younger ones, and is a preferred alternative to pharmacological therapy, particularly in patients with medical co-morbidities [5].

Evidence from meta-analytical studies suggests that ECT has a greater efficacy than with antidepressant medication [11]. This type of analysis has traditionally been administered using older medications and needs to be investigated using newer antidepressant medications that are available today. There are very few studies directly comparing acute and maintenance ECT (M-ECT) with pharmacological maintenance versus acute ECT and pharmacologicals alone (M-Pharm) over a substantial period.

Evidence indicates that the relapse rate of Major Depressive Disorders may be particularly high for older adults, with 50\% - 60\% experiencing a relapse within one year post-acute ECT while maintained on pharmacological treatment alone [12]. The sparse research studying M-ECT suggests that its incorporation in a maintenance pharmacological therapy can prevent patient relapse and readmission; however more research is needed, particularly into the benefits, and risks, 
of long-term M-ECT [13].

Several studies aimed at evaluating the benefits of M-ECT for Depressive Disorders in elderly patients lack adequate evidence. These studies are either case series, are retrospective in nature or follow the patients for an inadequate time [14]. Hence, prospective studies will add more power to the evidence of M-ECT treatment in elderly depressed patients.

Kellner et al. [5] did prospective randomized controlled studies in this population, though his studies had only a six month follow up. Further, the M-Pharm group was treated with older antidepressants, Nortryptaline and Lithium. This group is studied by comparison to a M-ECT group. Both interventions showed significantly lower relapse rates than historical placebo control groups. However, more than half of patients either experienced relapse of depression or dropped out of the study. Unfortunately, the patient drop-out rate is very high in pharmacological studies as a result of the extensive side-effects that patients experience on anti-depressant medication. Compliance in ECT therapy studies tend to be at a much higher rate as a result of substantially fewer negative effects on the patient. Multiple sources highlight the need for more studies investigating combined modalities of M-Pharm and concurrent M-ECT with extended follow-up periods.

In routine, daily clinical practice depressive disorders in the elderly are managed by psychosocial interventions and maintenance pharmacotherapy, typically consisting of SSRI or SNRI medication. Additional mood stabilizing medication and a typical antipsychotic medication may be used as a combination pharmacotherapy.

The purpose of this study is to determine prospectively the clinical outcomes of a 1-year long maintenance-ECT program using standardized measures of cognition, indices of quality of life, and psychometric indicators of depression. We hypothesized that 1) scores indicating severity of depression would be significantly reduced following the initiation of the M-ECT program; 2) quality of life indicators would increase as a result of on-going treatment; 3 ) overall cognition would be unchanged; and 4) that re-admissions into the hospital would be less.

\section{Methods and Materials}

Males and females over the age of 65 were recruited for this study. All participants included in the study had presented to the hospital (Health Sciences North) were diagnosed by consultant psychiatrists with moderate-to-severe major depressive disorder and were further referred for ECT as part of overall management of the treatment-resistance to medication alone; the patients were either inpatients or outpatients. These patients were subsequently followed by out-patient psychiatrists with maintenance pharmacotherapy which consisted of naturalistic follow-up in the community. Patients were excluded for the following reasons: 1) presence of major substance abuse disorders; 2) presence of co- 
ronary artery disease or cardiovascular stroke that had been diagnosed within the previous 6 months; 3 ) had already received ECT within the past two years. For this study, we identified both control and treatment groups on the basis of their clinical presentation. While the treatment population received M-ECT along with maintenance pharmacotherapy by their outpatient psychiatrists throughout the course of the study, individuals assigned to the control group did not by their own request; however to ensure equality and fairness participants assigned to both groups were all offered the opportunity for M-ECT. All participants were identified the study investigators (all Consultant Psychiatrists with Health Sciences North). All individuals in the treatment group were assessed by two psychiatrists and met criteria for M-ECT. Informed consent was obtained by the participant or by the substitute decision-making family member.

All participants were administered scales that were designed to measure severity of depression (Geriatric Depression Scale [GDS] and Hamilton Depression Scale [HDS]), cognition and memory problems (Mini Mental State Examination [MMSE] and the Montreal Cognitive Assessment [MoCA]), clinical function and daily functional activity (Clinical Global Impression Scale [CGI] and Functional Assessment Scale [FAS]), determination of burden of illness (Cumulative Illness Rating Scale-Geriatric [CIRS]), and overall quality of life [QOL]). All scales were given upon admission into the study (baseline) and at 6- and 12-months after the initial baseline for a total of 3 separate administrations of the tests. All statistical analysis was completed using SPSS v. 20.

Electroconvulsive therapy was administered with the aid of a nurse coordinator who monitored the entire process including lab work and pre-anaesthetic consultations. Before administration of ECT to the treatment group, two physicians approved and concluded that the patient was an ideal candidate for treatment. Each participant within the treatment group was admitted to HSN Sudbury into the Day Surgery Programme at approximately 6:30 AM and were investigated for temperature and other vital signs. They were taken to the ECT room where an anaesthetist administered propofol along with succinyl choline. ECT was administered by the psychiatrist using a MECTA 5000Q ECT machine using one of two patterns: an ultra brief pulse $0.3 \mathrm{~ms}$ or a brief pulse (between $0.5-1.0 \mathrm{~ms}$ ). All currents were maintained at $800 \mathrm{~mA}$. After the seizure had been verified, the anaesthetist engaged in recovery protocols and the participant remained under supervision until vital signs and indices of consciousness were stable. Finally, the nursing staff evaluated the patient for potential discharge and a psychiatrist reviewed the patient's health for evaluation of immediate side effects. The patient was then discharged from the day surgery program and an appointment was given for a follow-up with their outpatient psychiatrist. The patient was followed periodically in the out-patient clinic by their regular psychiatrists. The research assistant made appointments with the patient to collect the data.

All ethics was reviewed and approved by the Health Sciences North Research 
Ethics Board.

\section{Results}

A total of 29 participants elected to participate in the study (6 males and $23 \mathrm{fe}-$ males) with an average age of $68.52(\mathrm{SD}=7.03)$. There were a total of 12 participants in the treatment group and 17 participants in the control group, however only between 11 - 14 participants' data were available for the ensuing analyses due to hospital admission, relapse, study drop-out, or death due to natural causes.

Separate General Linear Models were employed to discern mean differences across the two treatment groups. The results of each analysis demonstrated no significant main effects or interactions for the following measures (sample size in parentheses): GDS $(\mathrm{N}-14)$, HDS $(\mathrm{N}=14)$, MMSE $(\mathrm{N}=14)$, MoCA $(\mathrm{N}=14)$, FAS $(\mathrm{N}=13)$, CIRS $(\mathrm{N}=14)$, QOL $(\mathrm{N}=14)$. One analysis did reveal a significant time by gender interaction between time and gender for the mean differences in the "Severity of Illness" component of the CGI ( $F=4.516, p=0.035$, partial eta $\left.{ }^{2}=0.49\right)$. Post-hoc examination of the data using paired t-tests indicated that mean CGI scores significantly decreased for females within the span of 1-year independent of group $(t=0.386, p=0.006$; Figure 1$)$.

On the basis of the observation that many participants in the control and treatment groups may have dropped-out as a result of their assigned group, we performed two Chi-Square analyses to determine proportions of those who had 1) completed the study $(\mathrm{N}=29)$ and 2) had been re-admitted to the hospital 1-year after completion of the study $(\mathrm{N}=12)$. The results revealed a significant disproportionality in attrition between the control and treatment groups $\left(X_{(1,29)}^{2}=\right.$ $5.40, \mathrm{p}=0.02$ ) whereby more patients assigned to the treatment group withdrew from the study; the analysis for readmissions was not significant $(\mathrm{p}=0.58)$.

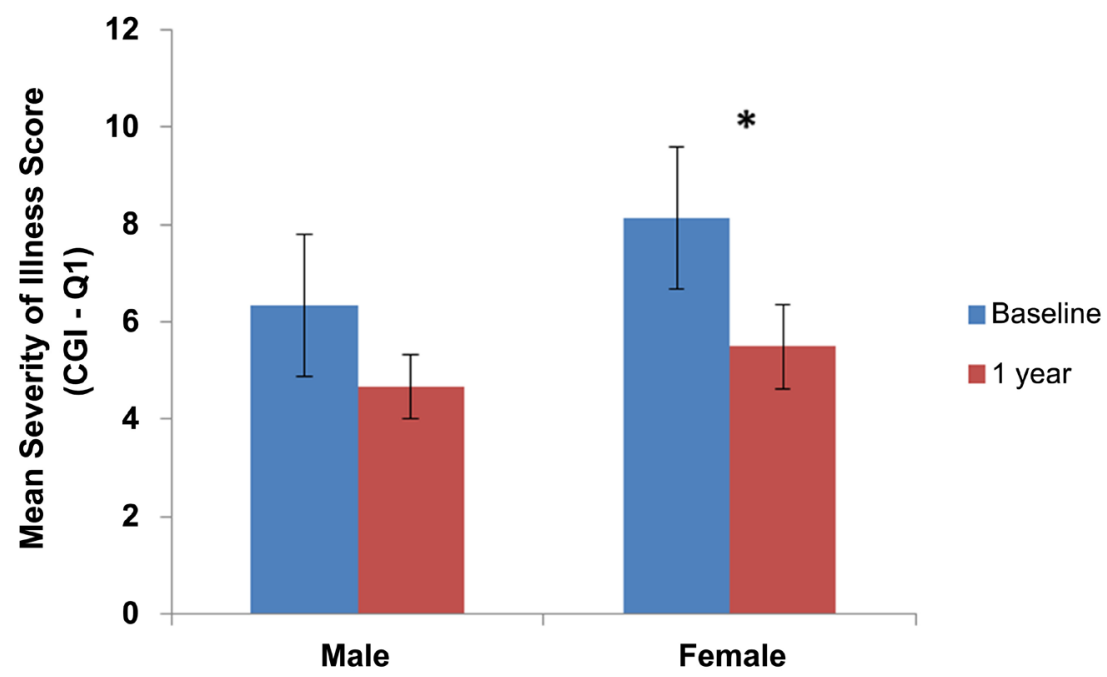

Figure 1. Significant time by gender interaction indicating that mean scores on the Severity of Ilness component of the CGI was significantly decreased for females 1-year later. Error bars represent standard error of the mean. 


\section{Discussion}

The present study was designed to assess the effects of maintenance ECT on elderly depressed people. The participants of the study were all 60 years of age or older and presented to the hospital with symptoms of major depressive disorder. During the course of the study there was a significant attrition rate because of voluntary withdrawal or death which may have limited the generalizeability of the findings; however the results obtained from this study indicated that administration of M-ECT did not significantly effect several quantitative measures of depressive symptoms, quality of life, or overall cognition or readmissions to the hospital.

This study consisted of individuals who were assigned to one of two groups: a control group comprised of individuals who refused M-ECT and a treatment group. While several previous studies have demonstrated a favourable response for elderly individuals who receive M-ECT as part of a treatment regimen for depressive disorders, many of the studies have lacked a control group as employed in this study. Provided that the sample size in each group was relatively low, our data indicated that there was no significant benefit for the administration of maintenance ECT when patients were followed-up at 6-month and 1 -year intervals as evidenced by similar scores on the various cognitive and psychiatric tests employed in the study as well as the insignificant difference in re-admissions for both the control and treatment groups and significant attrition by participants assigned to the treatment group. While we have no formal explanation for the attrition, we think that it may be associated with the inconsistency in the personnel involved in collecting the data.

A randomized controlled trial consisting of a much larger sample $(\mathrm{N}=201)$ assessed the efficacy of continuation ECT against a control group who were individuals administered lithium carbonate plus nortriptyline hydrochloride (C-Pharm) [5]. The results of this study indicated that both treatments were effective in limiting depressive relapse when compared against a placebo group as measured using the Hamilton Rating Scale for Depression; however the differences were not significant when both treatment groups were compared with each other. Our control group would have been comparable to their C-Pharm group because presumably the patients would have been receiving psychopharmacological therapy to mitigate their depressive symptoms. While this study consisted of individuals between the ages of 18 to 85 years of age and used continuation ECT (C-ECT) as the primary schedule for ECT therapy, our results support these findings. Similarly, a systematic review identified three randomized controlled trials, including the above-mentioned study [3]. It was concluded that in all three cases C-Pharm treatment was just as effective in reducing symptoms of depression as M-ECT. The authors also provided insight into the nature of the apparent similarities by citing the fact that regularized treatment schedules rather than individually-tailored schedules dependent upon the severity of symptoms may have diminished the possible benefits of providing ECT for 
the patients.

\section{Conclusion}

This prospective randomized study investigating the efficacy of M-ECT in elderly depressed individuals reveals that clinical outcomes are similar between individuals who opt to receive M-ECT and those who do not. There are clear limitations which shape the generalizability of this study to clinical contexts including availability of treatment regimens for both groups, possible population bias that include differences in overall willingness to receive M-ECT treatment and limited sample sizes. We contend that more thorough investigations including the addition of placebo groups would be advantageous to building the corpus of information required for clinicians to make informed decisions regarding the administration of M-ECT in elderly depressed populations.

\section{Acknowledgements}

This study was supported by a grant issued by the Northern Ontario Academic Medicine Association in Thunder Bay Canada.

\section{Conflicts of Interest}

The authors declare no conflicts of interest regarding the publication of this paper.

\section{References}

[1] Cole, M.G., Bellavance, F. and Mansour, A. (1999) Prognosis of Depression in Elderly Community and Primary Care Populations: A Systematic Review and Meta-Analysis. The American Journal of Psychiatry, 156, 1182-1189.

[2] Sackeim, H.A., Haskett, R.F., Mulsant, B.H., Thase, M.E., Mann, J.J., Pettinati, H. M. and Prudic, J. (2001) Continuation Pharmacotherapy in the Prevention of Relapse Following Electroconvulsive Therapy: A Randomized Controlled Trial. Journal of the American Medical Association, 285, 1299-1307. https://doi.org/10.1001/jama.285.10.1299

[3] Van Schaik, A.M., Comijs, H.C., Sonnenberg, C.M., Beekman, A.T., Sienaert, P. and Stek, M.L. (2012) Efficacy and Safety of Continuation and Maintenance Electroconvulsive Therapy in Depressed Elderly Patients: A Systematic Review. The American Journal of Geriatric Psychiatry, 20, 5-17. https://doi.org/10.1097/JGP.0b013e31820dcbf9

[4] Frederikse, M., Petrides, G. and Kellner, C. (2006) Continuation and Maintenance Electroconvulsive Therapy for the Treatment of Depressive Illness: A Response to the National Institute for Clinical Excellence Report. The Journal of ECT, 22, 13-17. https://doi.org/10.1097/00124509-200603000-00003

[5] Petrides, G., Tobias, K.G., Kellner, C.H. and Rudorfer, M.V. (2011) Continuation and Maintenance Electroconvulsive Therapy for Mood Disorders: Review of the Literature. Neuropsychobiology, 64, 129-140. https://doi.org/10.1159/000328943

[6] Kamat, S.M., Lefevre, P.J. and Grossberg, G.T. (2003) Electroconvulsive Therapy in the Elderly. Clinics in Geriatric Medicine, 19, 825-839.

https://doi.org/10.1016/S0749-0690(03)00043-0 
[7] Olfson, M., Marcus, S., Sackeim, H.A., Thompson, J. and Pincus, H.A. (1998) Use of ECT for the Inpatient Treatment of Recurrent Major Depression. American Journal of Psychiatry, 155, 22-29. https://doi.org/10.1176/ajp.155.1.22

[8] Tew Jr., J.D., Mulsant, B.H., Haskett, R.F., Prudic, J., Thase, M.E., Crowe, R.R. and Sackeim, H.A. (1999) Acute Efficacy of ECT in the Treatment of Major Depression in the Old-Old. American Journal of Psychiatry, 156, 1865-1870.

[9] O’Connor, D.W., Gardner, B., Presnell, I., Singh, D., Tsanglis, M. and White, E. (2010) The Effectiveness of Continuation-Maintenance ECT in Reducing Depressed Older Patients' Hospital Re-Admissions. Journal of Affective Disorders, 120, 62-66. https://doi.org/10.1016/j.jad.2009.04.005

[10] Thompson, J.W., Weiner, R.D. and Myers, C.P. (1994) Use of ECT in the United States in 1975, 1980, and 1986. American Journal of Psychiatry, 151, 1657-1661. https://doi.org/10.1176/ajp.151.11.1657

[11] Janicak, P.G., Davis, J.M., Gibbons, R.D., Ericksen, S., Chang, S. and Gallagher, P. (1985) Efficacy of ECT: A Meta-Analysis. The American Journal of Psychiatry, 142, 297-302.

[12] Huuhka, M., Korpisammal, L., Haataja, R. and Leinonen, E. (2004) One-Year Outcome of Elderly Inpatients with Major Depressive Disorder Treated with ECT and Antidepressants. The Journal of ECT, 20, 179-185.

https://doi.org/10.1097/00124509-200409000-00010

[13] Benbow, S.M. and Tench, D. (2007) A Survey of Psychiatrists in Northwest England Concerning Their Use of Maintenance Electroconvulsive Therapy. International Psychogeriatrics, 19, 985-987. https://doi.org/10.1017/S104161020700498X

[14] Stek, M.L., Wurff van der, F.F.B., Hoogendijk, W.J.G. and Beekman, A.T.F. (2003) Electroconvulsive Therapy for the Depressed Elderly. Cochrane Database of Systematic Reviews, No. 2, CD003593. https://doi.org/10.1002/14651858.CD003593 